\title{
Long-Term Aspirin Use and 5-Year Survival in Healthy Adults: A Population-Based Cohort Study in South Korea
}

\author{
Tak Kyu Oh and In-Ae Song \\ Department of Anesthesiology and Pain Medicine, Seoul National University Bundang Hospital, Seongnam, Korea.
}

Purpose: We investigated whether long-term aspirin use is associated with 5-year all-cause mortality.

Materials and Methods: Participants were individuals aged $\geq 40$ years who were registered in the 2010 sample cohort database of the National Health Insurance Service in South Korea. Aspirin users were divided into three groups: continuous users (20062010), previous users (2006-2009), and new users (2010). Individuals with a history of coronary artery disease and cerebrovascular disease were excluded. Five-year all-cause mortality was defined as mortality due to any cause from January 1, 2011 to December 31, 2015. Data were analyzed by multivariable Cox regression.

Results: In total, 424444 individuals were included. Five-year all-cause mortality was $9 \%$ lower in continuous aspirin users than in unexposed individuals [hazard ratio (HR): 0.91, 95\% confidence interval (CI): 0.86-0.97; $p=0.003$ ]. Five-year all-cause mortality rates in the new aspirin users (HR: 1.00, 95\% CI: 0.90-1.11; $p=0.995)$ and previous aspirin users (HR: 1.01, 95\% CI: 0.94-1.09; $p=0.776$ ) were not significantly different from that in unexposed individuals. In the 40-60-year age group, 5-year all-cause mortality in the continuous aspirin users was $24 \%$ lower (HR: 0.76, 95\% CI: 0.64-0.90; $p=0.002$ ) than that in unexposed individuals. However, in the $>60$-year age group, there was no significant association between aspirin use and 5-year all-cause mortality (HR: 0.96 , 95\% CI: $0.90-1.02 ; p=0.199$ ).

Conclusion: Long-term aspirin use is associated with reduced 5-year all-cause mortality in healthy adults, especially those aged $<60$ years.

Key Words: Aspirin, mortality, neoplasm, cohort study

\section{INTRODUCTION}

Aspirin, derived from salicylic acid, is one of the most frequently prescribed drugs worldwide for the primary and secondary prevention of cardiovascular diseases. ${ }^{1}$ A recent study reported that over $30 \%$ of adults aged $\geq 40$ years were prescribed as-

Received: June 16, 2020 Revised: October 21, 2020

Accepted: October 29, 2020

Corresponding author: In-Ae Song, MD, PhD, Department of Anesthesiology and Pain Medicine, Seoul National University Bundang Hospital, Gumi-ro 173beongil, Bundang-gu, Seongnam 13620, Korea.

Tel: 82-31-787-7499, Fax: 82-31-787-4063, E-mail: songoficu@outlook.kr

-The authors have no potential conflicts of interest to disclose.

(C) Copyright: Yonsei University College of Medicine 2020

This is an Open Access article distributed under the terms of the Creative Commons Attribution Non-Commercial License (https://creativecommons.org/licenses/ by-nc/4.0) which permits unrestricted non-commercial use, distribution, and reproduction in any medium, provided the original work is properly cited. pirin to prevent cardiovascular diseases. ${ }^{2}$ Despite its popularity, the usefulness of aspirin for the primary prevention of cardiovascular diseases remains controversial. ${ }^{3}$

Based on the evidence that aspirin use might prevent the development of both cancer and fatal cardiovascular events, ${ }^{4-8}$ it is expected that aspirin could improve mortality rates in healthy adults. A meta-analysis of aspirin use for the primary prevention of cardiovascular disease showed that it was beneficial for the prevention of all-cause mortality. ${ }^{9}$ However, a recent study, the Aspirin in Reducing Events in the Elderly (ASPREE) trial, reported that aspirin use did not prolong disability-free survival in healthy older adults; moreover, all-cause mortality was higher in apparently healthy older adults who received aspirin daily than in individuals in the placebo group. ${ }^{10,11}$ Additionally, cancer-related death was increased in the low-dose daily aspirin group. ${ }^{10}$ Although the ASPREE trial was a well-designed, large, population-based, randomized clinical trial, it comprised only 
healthy individuals aged 65 years or older. Thus, the effect of aspirin use on long-term mortality in the general adult population continues to be controversial and warrants further research. Accordingly, the aim of this population-based cohort study was to investigate whether long-term aspirin use is associated with 5 -year all-cause mortality in the adult population in South Korea.

\section{MATERIALS AND METHODS}

\section{Design and ethics statements}

This population-based cohort study was conducted according to the Reporting of Observational Studies in Epidemiology guidelines, ${ }^{12}$ and population, exposure, control, and outcomes were defined. ${ }^{13}$ The study protocol was approved by the Institutional Review Board (X-1903-529-903) and the Health Insurance Review and Assessment Service (NHIS-2019-2-170).

\section{Data source}

The National Health Insurance Service (NHIS) has developed a sample cohort database to provide data for academic healthcare-related research in the general population in South Korea. The database includes a stratified random sample of one million individuals registered with the agency since 2002. It was designed to be representative of the national population in terms of demographic and socioeconomic variables. The cohort was dynamic, and the patients were followed up until the end of 2015. These data were supplemented with additional data, including data on infants, to make-up for attrition due to follow-up loss and death. ${ }^{14}$

\section{Study population}

Individuals of the general adult population (aged $\geq 40$ years) registered in the 2010 cohort database of the NHIS were included in this study. Individuals who died in 2010 and those who had a history of cerebrovascular or coronary artery diseases were excluded from analysis because we focused on the primary preventive effect of aspirin on 5-year mortality. These exclusions were performed based on the registered International Statistical Classification of Diseases (ICD)-10 codes from 2009 to 2010 in the NHIS database, such as I21*, I22*, and I25.2* for coronary artery disease and G45*, G46*, H34.0, and I60*-I69* for cerebrovascular disease.

\section{Main exposure variable: aspirin use}

To define aspirin exposure, prescription data for aspirin for 2006-2010 (5 years) were extracted. Using these prescription data, we defined individuals who had been prescribed aspirin for $>30$ days regularly and continuously as aspirin users. The aspirin users were divided into three groups (continuous, previous, and new aspirin users). Continuous aspirin users were prescribed to take aspirin continuously for 5 years from 2006 to 2010. Previous aspirin users were defined as individuals who were prescribed aspirin from 2006 to 2009, but did not use aspirin in 2010. New aspirin users were defined as individuals who were newly prescribed to take aspirin in 2010, but were not prescribed aspirin from 2006 to 2009. In addition, individuals who used aspirin from 2007 and 2008 to 2010 continuously were classified as continuous aspirin users, whereas those who used aspirin from 2009 to 2010 were classified as new aspirin users.

\section{Reference population of unexposed individuals}

The reference population (unexposed individuals) in this study comprised individuals who did not use aspirin from 2006 to 2010 among the population in the 2010 NHIS cohort.

\section{Study endpoints}

The primary endpoint of this study was 5-year all-cause mortality, and the secondary endpoints were 5-year cardiovascular mortality and cancer mortality. Five-year all-cause mortality was defined as mortality from any cause between January 1 , 2011 and December 31, 2015. Five-year cardiovascular mortality was defined as mortality due to a cardiovascular disease (ICD-10: I00-I99, Q20-Q28), and 5-year cancer mortality was defined as mortality due to cancer (ICD-10: C00-D48).

\section{Confounders}

Data on the following variables were collected as confounders in this study: 1) demographic information (age and sex); 2) annual income level (0-20\%, 20-40\%, 40-60\%, 60-80\%, and 80$100 \%$ ); 3) residence in 2010 (capital city, metropolitan city, and others); 4) the Charlson comorbidity index (CCI), which was calculated based on ICD-10 codes from 2009 to 2010, as shown in Supplementary Material (only online); 5) comorbidities that were registered using ICD-10 codes from 2009 to 2010; 6) exposure to clopidogrel; and 7) number of outpatient clinic visits in 2010 (days). Individuals in the cohort who were prescribed regular clopidogrel in 2010 were considered exposed to clopidogrel.

\section{Statistical analysis}

The baseline characteristics are presented as means with standard deviations for continuous variables and numbers with percentages for categorical variables. We fitted the multivariable Cox regression model for 5-year all-cause mortality to the entire cohort. All covariates were included in the multivariable model for adjustment, except CCI, to avoid multicollinearity with individual underlying diseases used to calculate CCI. In the multivariable model, the risk of 5-year all-cause mortality in continuous, previous, and new aspirin users was investigated in comparison to that in the unexposed individuals. We then performed a competing risk analysis of 5-year cardiovascular mortality and 5-year cancer mortality using the same methods of the main analysis (multivariable Cox regression model). For these competing risk analyses, death due to cardiovascular disease or cancer was set as an event in the Cox model, and the duration 
from January 1, 2011 to the date of death was used as the time to event. We performed a subgroup analysis according to age (>60-year and 40-60-year age groups). All covariates were included in the subgroup analyses for multivariable adjustment.

To enhance the robustness of our main findings, we performed propensity score (PS) matching, which is known to reduce confounders in cohort studies, ${ }^{15}$ using the nearest neighbor method with a 1:3 ratio, without replacement, and a caliper width of 0.1 . The continuous aspirin users and unexposed individuals were included in this PS modeling, not new aspirin users or previous aspirin users. A logistic regression analysis was performed to calculate the PS values as a logistic model, and all covariates were included in the PS model. The absolute value of the standardized mean difference (ASD) was used to determine the balance between the aspirin and control groups before and after PS matching. The ASDs between the two groups before and after PS matching were set to $<0.1$ to determine if the two groups were well balanced through PS matching. In addition, we used C-statistics as a measure of the ability of the PS to control confounding. ${ }^{16}$ After confirming good balance between the two groups (continuous aspirin users and the unexposed individuals), we performed a stratified Cox regression analysis of 5-year all-cause mortality in the PS-matched cohort.

The results of the Cox regression models are presented as hazard ratios (HRs) with $95 \%$ confidence intervals (CIs); it was confirmed that there was no multicollinearity in all multivariable models of the entire cohort with a variance inflation factor $<2.0$. C-statistics were used to identify the C-index of the multivariable Cox regression models. All statistical analyses were performed using R version 3.6.3 (R Foundation for Statistical Computing, Vienna, Austria), and results with $p<0.05$ were considered statistically significant.

\section{RESULTS}

The 2010 NHIS sample cohort comprised 826909 individuals. Of these, we excluded 4487 individuals who died in 2010. Furthermore, 338471 individuals who were $<40$ years of age and 59507 individuals who had a history of cerebrovascular or coronary artery diseases were excluded from the analysis. Finally, 424444 individuals were included in the analysis. Of these, 16447 individuals (3.9\%) died within 5 years, among which 2864 ( $0.7 \%)$ and $5707(1.3 \%)$ deaths corresponded to (5-year) cardiovascular and cancer mortality, respectively (Fig. 1). The baseline characteristics of the study subjects are presented in Table 1. Among the individuals, the numbers of continuous aspirin users, previous aspirin users, and new aspirin users were $18482(4.4 \%)$, $9332(2.2 \%)$, and $5311(1.1 \%)$, respectively.

\section{Survival analysis for the entire cohort}

Table 2 shows the results of the survival analysis for the entire cohort. The 5 -year all-cause mortality was $9 \%$ lower in the con-

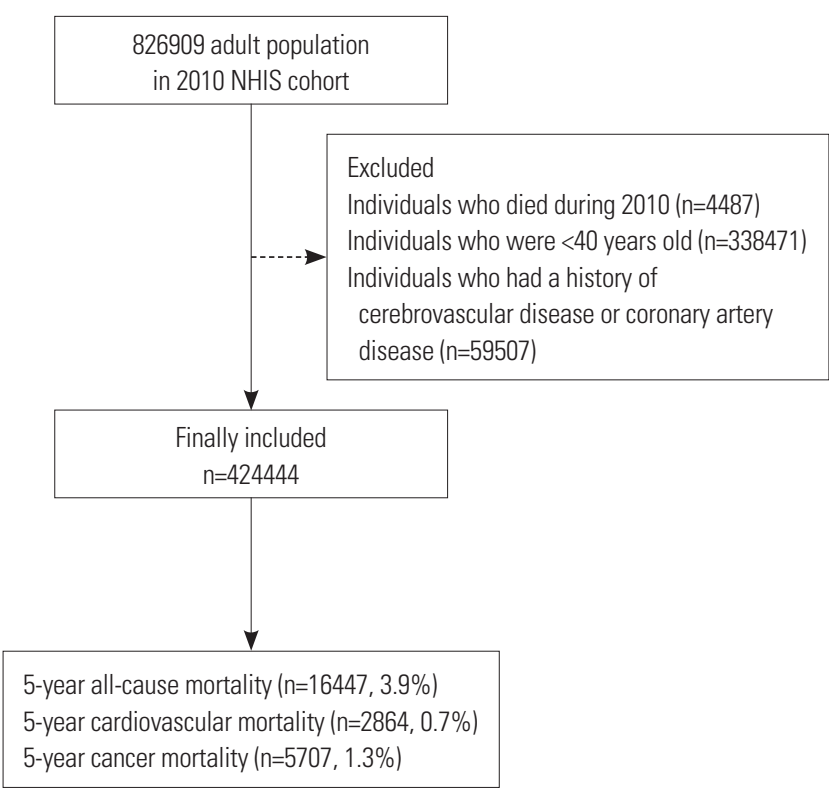

Fig. 1. Flow chart showing the participant selection process. NHIS, National Health Insurance Service.

tinuous aspirin users than in unexposed individuals (HR: 0.91, 95\% CI: 0.86-0.97; $p=0.003$ ). However, 5 -year all-cause mortality in new aspirin users (HR: 1.00, 95\% CI: 0.90-1.11; $p=0.995$ ) and previous aspirin users (HR: 1.01, 95\% CI: $0.94-1.09 ; p=0.776$ ) was not significantly different from that in the unexposed individuals. The C-index of the multivariable model was 0.81 (95\% CI: 0.80-0.81).

Table 3 shows the results of competing risk analysis of 5 -year cancer and cardiovascular mortality. The 5 -year cancer mortality in continuous aspirin users was $14 \%$ lower (HR: $0.86,95 \%$ CI: $0.77-0.96 ; p=0.002$ ) than that in unexposed individuals. However, 5-year cardiovascular mortality in the continuous aspirin users did not show a significant association (HR: 1.01, 95\% CI: 0.88-1.15; $p=0.940$ ), compared to that in unexposed individuals.

Table 4 shows the results of subgroup analysis of 5-year allcause mortality according to age. In the $>60$-year age group ( $\mathrm{n}=$ 111072), there was no significant association between 5 -year all-cause mortality and aspirin use (HR: 0.96, 95\% CI: 0.90-1.02; $p=0.199$ ). However, in the $40-60$-year age group, 5 -year all-cause mortality in the continuous aspirin users was $24 \%$ lower (HR: $0.76,95 \%$ CI: $0.64-0.90 ; p=0.002)$ than that in the unexposed individuals.

\section{Survival analysis after propensity score matching}

The characteristics of the continuous aspirin users and unexposed individuals before and after PS matching are presented in Supplementary Table 1 (only online). All covariates were well balanced, as indicated by their ASDs, which were below 0.1 , and the C-index of the PS model was 0.83 (95\% CI: 0.82-0.83). Table 5 shows the results of the survival analysis after PS matching. After PS matching, the 5-year all-cause mortality in the continuous aspirin users was $7.0 \%$ (1289/18482), whereas that in 
Table 1. Baseline Characteristics of the Participants $(n=424444)$

\begin{tabular}{|c|c|c|}
\hline Variable & $\mathbf{n}(\%)$ & Mean (SD) \\
\hline Age (yr) & & $54.3(10.9)$ \\
\hline Sex: male (vs. female) & 205021 (48.3) & \\
\hline \multicolumn{3}{|l|}{ Income level } \\
\hline 0-20\% (lowest) & $60128(14.2)$ & \\
\hline $20-40 \%$ & $62016(14.6)$ & \\
\hline $40-60 \%$ & $87134(20.5)$ & \\
\hline $60-80 \%$ & $89719(21.1)$ & \\
\hline 80-100\% (highest) & $125447(29.6)$ & \\
\hline \multicolumn{3}{|l|}{ Residence } \\
\hline Capital city (Seoul) & $85872(20.2)$ & \\
\hline Metropolitan city & $109186(25.7)$ & \\
\hline Other area & $229386(54.0)$ & \\
\hline Charlson comorbidity index & & $1.3(1.7)$ \\
\hline Hypertension & $100095(23.6)$ & \\
\hline DM with chronic complication & 20594 (4.9) & \\
\hline DM without chronic complication & $55429(13.1)$ & \\
\hline Congestive heart failure & $7725(1.8)$ & \\
\hline Chronic pulmonary disease & $111465(26.3)$ & \\
\hline Dementia & $825(0.2)$ & \\
\hline Hemi- or paraplegia & $1145(0.3)$ & \\
\hline Renal disease & $2911(0.7)$ & \\
\hline Mild liver disease & $81239(19.1)$ & \\
\hline Severe liver disease & $3557(0.8)$ & \\
\hline Peptic ulcer disease & 117864 (27.8) & \\
\hline Rheumatic disease & $16721(3.9)$ & \\
\hline Peripheral vascular disease & 42086 (9.9) & \\
\hline Clopidogrel user in 2010 & $1166(0.3)$ & \\
\hline \multicolumn{3}{|l|}{ Outpatient clinic visit in 2010 , day } \\
\hline $0-7$ & $224272(52.8)$ & \\
\hline $8-30$ & $176367(41.6)$ & \\
\hline 30-90 & $23390(5.5)$ & \\
\hline$>90$ & $415(0.1)$ & \\
\hline \multicolumn{3}{|l|}{ Aspirin users } \\
\hline Unexposed individuals & 391319 (92.2) & \\
\hline New aspirin users (2010) & $5311(1.3)$ & \\
\hline Previous aspirin users (2006-2009) & $9332(2.2)$ & \\
\hline Continuous aspirin users (2006-2010) & $18482(4.4)$ & \\
\hline
\end{tabular}

SD, standard deviation; DM, diabetes mellitus.

Presented as numbers with percentages or means with standard deviations.

the unexposed individuals was $7.5 \%$ (4046/53675). We also found that the 5 -year all-cause mortality was $7 \%$ lower in the continuous aspirin users (HR: $0.93,95 \%$ CI: $0.87-0.98 ; p=0.014$ ) than in the unexposed individuals.

\section{DISCUSSION}

The results of this population-based cohort study showed that long-term aspirin use is associated with reduced 5-year all-cause
Table 2. Multivariable Cox Regression Model for 5-Year All-Cause Mortality in the 2010 NHIS Sample Cohort

\begin{tabular}{|c|c|c|}
\hline \multirow{2}{*}{ Variable } & \multirow{2}{*}{$\frac{\text { Multivariable model }}{\text { HR }(95 \% \text { CI) }}$} & \multirow{2}{*}{$p$ value } \\
\hline & & \\
\hline Age (yr) & $1.11(1.10-1.11)$ & $<0.001$ \\
\hline Sex: male (vs. female) & $1.97(1.91-2.04)$ & $<0.001$ \\
\hline \multicolumn{3}{|l|}{ Income level } \\
\hline 0-20\% (lowest) & 1 & \\
\hline $20-40 \%$ & $0.99(0.93-1.05)$ & 0.647 \\
\hline $40-60 \%$ & $1.08(1.02-1.13)$ & 0.004 \\
\hline $60-80 \%$ & $0.84(0.80-0.88)$ & $<0.001$ \\
\hline 80-100\% (highest) & $0.73(0.69-0.76)$ & $<0.001$ \\
\hline \multicolumn{3}{|l|}{ Residence } \\
\hline Capital city (Seoul) & 1 & \\
\hline Metropolitan city & $1.19(1.13-1.25)$ & $<0.001$ \\
\hline Other area & $1.16(1.11-1.21)$ & $<0.001$ \\
\hline \multicolumn{3}{|l|}{ Comorbidities } \\
\hline Hypertension & $1.04(1.00-1.08)$ & 0.062 \\
\hline DM with chronic complication & $1.34(1.27-1.41)$ & $<0.001$ \\
\hline DM without chronic complication & $1.27(1.22-1.32)$ & $<0.001$ \\
\hline Congestive heart failure & $1.59(1.50-1.69)$ & $<0.001$ \\
\hline Chronic pulmonary disease & $1.10(1.06-1.14)$ & $<0.001$ \\
\hline Dementia & $2.06(1.81-2.35)$ & $<0.001$ \\
\hline Hemi- or paraplegia & $2.38(2.08-2.73)$ & $<0.001$ \\
\hline Renal disease & $2.00(1.82-2.19)$ & $<0.001$ \\
\hline Mild liver disease & $1.16(1.12-1.20)$ & $<0.001$ \\
\hline Severe liver disease & $2.59(2.37-2.84)$ & $<0.001$ \\
\hline Peptic ulcer disease & $0.96(0.93-1.00)$ & 0.027 \\
\hline Rheumatic disease & $1.03(0.96-1.10)$ & 0.432 \\
\hline Peripheral vascular disease & $0.94(0.93-1.10)$ & 0.027 \\
\hline Clopidogrel user in 2010 & $1.06(0.88-1.28)$ & 0.527 \\
\hline \multicolumn{3}{|l|}{ Outpatient clinic visit in 2010 , day } \\
\hline $0-7$ & 1 & \\
\hline $8-30$ & $0.69(0.66-0.71)$ & $<0.001$ \\
\hline 30-90 & $0.65(0.62-0.69)$ & $<0.001$ \\
\hline$>90$ & $0.83(0.65-1.06)$ & 0.137 \\
\hline \multicolumn{3}{|l|}{ Aspirin users } \\
\hline Unexposed individuals & 1 & \\
\hline New aspirin users (2010) & $1.00(0.90-1.11)$ & 0.995 \\
\hline Previous aspirin users (2006-2009) & $1.01(0.94-1.09)$ & 0.776 \\
\hline Continuous aspirin users (2006-2010) & $0.91(0.86-0.97)$ & 0.003 \\
\hline
\end{tabular}

NHIS, National Health Insurance Service; HR, hazard ratio; $\mathrm{Cl}$, confidence interval; DM, diabetes mellitus.

C-index: 0.81 (95\% Cl: 0.80-0.81).

mortality in the adult population in South Korea. This association was more evident in aspirin users aged $<60$ years. Additionally, the results of competing risk analysis suggested that 5 -year cancer mortality, but not cardiovascular mortality, might be reduced in continuous aspirin users. This result was similar to that of a recent cohort study, which reported that aspirin use is associated with a reduction in all-cause and cancer mortality. ${ }^{17}$

The results of this observational study should be interpreted 
carefully. In this study, only continuous aspirin use was associated with a benefit in terms of 5-year all-cause and cancer mortality, compared to that for unexposed individuals. However, the comparison between the continuous aspirin users and unexposed individuals has some limitations. First, if the use of aspirin affects mortality shortly after treatment initiation, this is not captured in an analysis of continuous users. In addition, the

Table 3. Competing Risk Analyses of 5-Year Cancer Mortality and Cardiovascular Mortality

\begin{tabular}{lcc}
\hline \multicolumn{1}{c}{ Variable } & Multivariable model & \\
\cline { 2 - 2 } & HR value \\
\hline 5-year cancer mortality & & \\
$\quad$ Unexposed individuals & & \\
\hline New aspirin users (2010) & $0.98(0.81-1.18)$ & 0.833 \\
\hline Previous aspirin users (2006-2009) & $0.88(0.76-1.02)$ & 0.079 \\
\hline Continuous aspirin users (2006-2010) & $0.86(0.77-0.96)$ & 0.002 \\
\hline 5-year cardiovascular mortality & & \\
\hline Unexposed individuals & 1 & \\
\hline New aspirin users (2010) & $1.25(1.01-1.57)$ & 0.052 \\
\hline Previous aspirin users (2006-2009) & $1.18(1.00-1.40)$ & 0.051 \\
\hline Continuous aspirin users (2006-2010) & $1.01(0.88-1.15)$ & 0.940 \\
\hline
\end{tabular}

$\mathrm{HR}$, hazard ratio; $\mathrm{Cl}$, confidence interval.

Table 4. Subgroup Analyses of 5-Year All-Cause Mortality According to Age

\begin{tabular}{|c|c|c|}
\hline \multirow{2}{*}{ Variable } & Multivariable model & \multirow{2}{*}{$p$ value } \\
\hline & HR $(95 \%$ Cl) & \\
\hline \multicolumn{3}{|l|}{ Age $>60, n=111072$} \\
\hline Unexposed individuals & 1 & \\
\hline New aspirin users (2010) & $1.00(0.88-1.12)$ & 0.929 \\
\hline Previous aspirin users (2006-2009) & $1.04(0.96-1.14)$ & 0.324 \\
\hline Continuous aspirin users (2006-2010) & $0.96(0.90-1.02)$ & 0.199 \\
\hline \multicolumn{3}{|l|}{ Age $40-60, n=313372$} \\
\hline Unexposed individuals & 1 & \\
\hline New aspirin users (2010) & $1.12(0.89-1.42)$ & 0.335 \\
\hline Previous aspirin users (2006-2009) & $0.87(0.71-1.08)$ & 0.199 \\
\hline Continuous aspirin users (2006-2010) & $0.76(0.64-0.90)$ & 0.002 \\
\hline
\end{tabular}

$\mathrm{HR}$, hazard ratio; $\mathrm{Cl}$, confidence interval. continuous aspirin users tended to be healthy adherers and represented those who would benefit from treatment, which could also affect the results. Compared to the continuous aspirin users, the new users or previous aspirin users might not be exhibiting good treatment adherence or the duration of aspirin exposure might not have been sufficient to yield a clinical benefit with respect to long-term mortality.

Owing to this being an observational study, indication bias should also be considered. ${ }^{18}$ In this study, aspirin might have been prescribed for the primary prevention of acute cardiovascular events in the healthy population without a history of cerebrovascular or coronary artery disease. However, the aspirin users in this study of older age or with higher CCI scores might have had a higher risk of acute cardiovascular events as shown in Supplementary Table 1 (only online). Although we controlled for confounders using multivariable adjustments and PS matching, the indication of aspirin might affect the results. For example, the association between 5-year cardiovascular mortality and aspirin use was not significant in the aspirin users, compared with that in unexposed individuals. However, considering indication bias, the results could have been influenced by the characteristics of aspirin users and not by the effect of aspirin itself.

The results of the present study are different from those of the ASPREE trial, ${ }^{10,11,19}$ which reported higher all-cause mortality in the aspirin group than in the placebo group. As the ASPREE trial focused on healthy older individuals with a lower risk of mortality than older individuals who had chronic diseases, aspirin use was expected to prevent primary cardiovascular events and cancer development and not secondary events. In the ARRIVE trial, the primary prevention of cardiovascular events in aspirin users was not significant (HR: 0.96, 95\% CI: 0.81-1.13; $p=0.60$ ) among 12546 men (aged $\geq 55$ years) and women (aged $\geq 60$ years) with moderate cardiovascular disease risk. ${ }^{20}$ However, gastrointestinal bleeding was higher in participants who received aspirin [odds ratio (OR): $2.11,95 \% \mathrm{CI}$ : 1.36-3.28; $p=$ 0.007 ], as a major complication of aspirin use. ${ }^{21}$ In another recent trial of cardiovascular events in diabetes conducted with 15480 participants, the fatal vascular death rate did not differ

Table 5. Five-Year Mortality after PS Matching

\begin{tabular}{|c|c|c|c|}
\hline Variables & Mortality (n, \%) & HR (95\% CI) & $p$ value \\
\hline \multicolumn{4}{|l|}{ After PS matching } \\
\hline \multicolumn{4}{|l|}{ 5-year all-cause mortality } \\
\hline Unexposed individuals & 4046 of $53675(7.5)$ & 1 & \\
\hline Continuous aspirin users & 1289 of $18482(7.0)$ & $0.93(0.87-0.98)$ & 0.014 \\
\hline \multicolumn{4}{|l|}{ 5-year cancer mortality } \\
\hline Unexposed individuals & 1270 of $53675(2.4)$ & 1 & \\
\hline Continuous aspirin users & 406 of $18482(2.2)$ & $0.93(0.83-1.04)$ & 0.175 \\
\hline \multicolumn{4}{|l|}{ 5-year cardiovascular mortality } \\
\hline Unexposed individuals & 800 of 53675 (1.5) & 1 & \\
\hline Continuous aspirin users & 283 of 18482 (1.5) & $1.02(0.89-1.17)$ & 0.731 \\
\hline
\end{tabular}

PS, propensity score; $\mathrm{HR}$, hazard ratio; $\mathrm{Cl}$, confidence interval. 
between the aspirin and placebo groups, and increased major bleeding (OR: 1.29, 95\% CI: 1.09-1.52; $p=0.003$ ) was reported in the aspirin group..$^{22}$ Furthermore, a recent meta-analysis concluded that aspirin was not associated with a reduction in the incidence of all-cause mortality; however, it was associated with an increased incidence of major bleeding. ${ }^{23}$ The authors of the meta-analysis suggested that the routine use of aspirin for primary prevention should be reconsidered. Considering that the 5 -year cardiovascular mortality in our competing risk analysis was not significantly associated with aspirin use, compared to that for unexposed individuals, the effect of aspirin use on the prevention of cardiovascular events might not be significant, as reported in previous clinical trials. ${ }^{19,21-23}$

Although our study was not a randomized clinical trial, we covered a much broader population, including the general adult population aged 40 years or above, who had no history of cerebrovascular or coronary artery diseases. Thus, the association with aspirin use might be applied to the primary prevention of cardiovascular disease or cancer. Additionally, we included many confounders in this analysis, such as comorbidities related to cardiovascular disease-related risk factors, frailty-related information, and socioeconomic status-related information. Therefore, the residual confounders were minimized in this study, despite the limitations of the study design.

The results of the competing risk analysis in our study were noteworthy. The association between exposure to aspirin and an improvement in 5-year mortality was more evident with respect to death due to cancer. A recent population-based cohort study, which was conducted in Hong Kong, reported that the long-term use of low-dose aspirin was associated with a decreased risk of cancer, except for breast cancer. ${ }^{24}$ Other population-based cohort studies in South Korea and the United States have also reported that aspirin use was associated with a decreased risk of lung cancer. ${ }^{25,26}$ However, another recent cohort study reported that low-dose aspirin use did not reduce cancer incidence and cancer mortality in older individuals (aged 6085 years) in Japan. ${ }^{27}$ Similarly, another population-based cohort study conducted on the Danish population reported that ovarian cancer mortality was not associated with aspirin use. ${ }^{28}$ Thus, the relationship between the use of aspirin and risk of cancer or cancer mortality remains controversial.

The results of subgroup analyses indicated that the association between aspirin use and reduced 5-year all-cause mortality is more evident in individuals aged $40-60$ years, not in those $>60$ years. This suggests that the effect of aspirin on reduced 5 -year all-cause mortality is applicable to younger individuals and not older individuals. A previous study of the ASPREE trial focusing on older adults (70 years or older) reported that higher mortality was observed in the aspirin group than in the placebo group, and this is in contrast to the findings of our study. ${ }^{10}$ Other studies also failed to demonstrate the effect of aspirin on primary prevention for serious and adverse cardiovascular events in older individuals. ${ }^{20,22}$ Therefore, the primary preven- tive effect of aspirin remains controversial, especially in older individuals, ${ }^{29}$ and the results of the subgroup analyses validated this.

This study had some limitations. First, some important variables, such as body mass index, were not included in the statistical adjustment, because they were not included in the NHIS dataset. Second, we defined comorbidities using the ICD-10 codes registered in the NHIS database. The diseases specified by the ICD-10 codes might differ from the actual underlying diseases in all patients. Third, PS adjustment and multivariable adjustment could control for only known confounders; thus, there might be residual confounders that could affect the study results. Fourth, we based the analysis on aspirin prescription data and did not assess adherence or compliance in those classified as aspirin users. Lastly, indication bias might have affected the results of this study, as we previously mentioned.

In conclusion, the results of this population-based cohort study suggested that long-term aspirin use is associated with reduced 5-year all-cause mortality in the general population of adults ( $\geq 40$ years) with no history of cerebrovascular or coronary artery disease. This association was more evident in individuals aged $<60$ years and for 5 -year cancer mortality. Therefore, the effect of aspirin use on long-term mortality remains controversial, and further studies are needed to clarify this issue.

\section{AUTHOR CONTRIBUTIONS}

Conceptualization: Tak Kyu Oh and In-Ae Song. Data curation: Tak Kyu Oh. Formal analysis: Tak Kyu Oh. Investigation: In-Ae Song. Methodology: Tak Kyu Oh and In-Ae Song. Project administration: Tak Kyu Oh and In-Ae Song. Resources: Tak Kyu Oh and In-Ae Song. Supervision: In-Ae Song. Validation: Tak Kyu Oh and In-Ae Song. Visualization: Tak Kyu Oh and In-Ae Song. Writing_original draft: Tak Kyu Oh. Writing_-review \& editing: In-Ae Song. Approval of final manuscript: Tak Kyu Oh and In-Ae Song.

\section{ORCID iDs}

Tak Kyu Oh https://orcid.org/0000-0002-4027-4423 In-Ae Song https://orcid.org/0000-0002-7814-4253

\section{REFERENCES}

1. Raber I, McCarthy CP, Vaduganathan M, Bhatt DL, Wood DA, Cleland JGF, et al. The rise and fall of aspirin in the primary prevention of cardiovascular disease. Lancet 2019;393:2155-67.

2. Stuntz M, Bernstein B. Recent trends in the prevalence of low-dose aspirin use for primary and secondary prevention of cardiovascular disease in the United States, 2012-2015. Prev Med Rep 2016;5: 183-6.

3. Raju NC, Eikelboom JW. The aspirin controversy in primary prevention. Curr Opin Cardiol 2012;27:499-507.

4. Rothwell PM. Aspirin in prevention of sporadic colorectal cancer: current clinical evidence and overall balance of risks and benefits. Recent Results Cancer Res 2013;191:121-42.

5. Rothwell PM, Price JF, Fowkes FG, Zanchetti A, Roncaglioni MC, 
Tognoni G, et al. Short-term effects of daily aspirin on cancer incidence, mortality, and non-vascular death: analysis of the time course of risks and benefits in 51 randomised controlled trials. Lancet 2012; 379:1602-12.

6. Sutcliffe P, Connock M, Gurung T, Freeman K, Johnson S, Kandala NB, et al. Aspirin for prophylactic use in the primary prevention of cardiovascular disease and cancer: a systematic review and overview of reviews. Health Technol Assess 2013;17:1-253.

7. Cao Y, Nishihara R, Wu K, Wang M, Ogino S, Willett WC, et al. Population-wide impact of long-term use of aspirin and the risk for cancer. JAMA Oncol 2016;2:762-9.

8. Sandler RS, Halabi S, Baron JA, Budinger S, Paskett E, Keresztes R, et al. A randomized trial of aspirin to prevent colorectal adenomas in patients with previous colorectal cancer. N Engl J Med 2003; 348:883-90.

9. Raju N, Sobieraj-Teague M, Hirsh J, O’Donnell M, Eikelboom J. Effect of aspirin on mortality in the primary prevention of cardiovascular disease. Am J Med 2011;124:621-9.

10. McNeil JJ, Nelson MR, Woods RL, Lockery JE, Wolfe R, Reid CM, et al. Effect of aspirin on all-cause mortality in the healthy elderly. N Engl J Med 2018;379:1519-28.

11. McNeil JJ, Woods RL, Nelson MR, Reid CM, Kirpach B, Wolfe R, et al. Effect of aspirin on disability-free survival in the healthy elderly. N Engl J Med 2018;379:1499-508.

12. Vandenbroucke JP, von Elm E, Altman DG, Gøtzsche PC, Mulrow $\mathrm{CD}$, Pocock SJ, et al. Strengthening the reporting of observational studies in epidemiology (STROBE): explanation and elaboration. PLoS Med 2007;4:e297.

13. Morgan RL, Whaley P, Thayer KA, Schünemann HJ. Identifying the PECO: a framework for formulating good questions to explore the association of environmental and other exposures with health outcomes. Environ Int 2018;121:1027-31.

14. Lee J, Lee JS, Park SH, Shin SA, Kim K. Cohort Profile: The National Health Insurance Service-National Sample Cohort (NHISNSC), South Korea. Int J Epidemiol 2017;46:e15.

15. Rosenbaum PR, Rubin DB. Reducing bias in observational studies using subclassification on the propensity score. J Am Stat Assoc 1984;79:516-24.

16. Westreich D, Cole SR, Funk MJ, Brookhart MA, Stürmer T. The role of the c-statistic in variable selection for propensity score models. Pharmacoepidemiol Drug Saf 2011;20:317-20.

17. Loomans-Kropp HA, Pinsky P, Cao Y, Chan AT, Umar A. Association of aspirin use with mortality risk among older adult partici- pants in the prostate, lung, colorectal, and ovarian cancer screening trial. JAMA Netw Open 2019;2:e1916729.

18. Kyriacou DN, Lewis RJ. Confounding by indication in clinical research. JAMA 2016;316:1818-9.

19. McNeil JJ, Wolfe R, Woods RL, Tonkin AM, Donnan GA, Nelson $\mathrm{MR}$, et al. Effect of aspirin on cardiovascular events and bleeding in the healthy elderly. N Engl J Med 2018;379:1509-18.

20. Gaziano JM, Brotons C, Coppolecchia R, Cricelli C, Darius H, Gorelick $\mathrm{PB}$, et al. Use of aspirin to reduce risk of initial vascular events in patients at moderate risk of cardiovascular disease (ARRIVE): a randomised, double-blind, placebo-controlled trial. Lancet 2018; 392:1036-46.

21. Silagy CA, McNeil JJ, Donnan GA, Tonkin AM, Worsam B, Campion K. Adverse effects of low-dose aspirin in a healthy elderly population. Clin Pharmacol Ther 1993;54:84-9.

22. ASCEND Study Collaborative Group, Bowman L, Mafham M, Wallendszus K, Stevens W, Buck G, et al. Effects of aspirin for primary prevention in persons with diabetes mellitus. N Engl J Med 2018; 379:1529-39.

23. Mahmoud AN, Gad MM, Elgendy AY, Elgendy IY, Bavry AA. Efficacy and safety of aspirin for primary prevention of cardiovascular events: a meta-analysis and trial sequential analysis of randomized controlled trials. Eur Heart J 2019;40:607-17.

24. Tsoi KKF, Ho JMW, Chan FCH, Sung JJY. Long-term use of lowdose aspirin for cancer prevention: a 10-year population cohort study in Hong Kong. Int J Cancer 2019;145:267-73.

25. Slatore CG, Au DH, Littman AJ, Satia JA, White E. Association of nonsteroidal anti-inflammatory drugs with lung cancer: results from a large cohort study. Cancer Epidemiol Biomarkers Prev 2009; 18:1203-7.

26. Ye S, Lee M, Lee D, Ha EH, Chun EM. Association of long-term use of low-dose aspirin as chemoprevention with risk of lung cancer. JAMA Netw Open 2019;2:e190185.

27. Yokoyama K, Ishizuka N, Uemura N, Mizokami Y, Hiraishi H, Murata $\mathrm{M}$, et al. Effects of daily aspirin on cancer incidence and mortality in the elderly Japanese. Res Pract Thromb Haemost 2018;2: 274-81.

28. Verdoodt F, Kjaer SK, Dehlendorff C, Friis S. Aspirin use and ovarian cancer mortality in a Danish nationwide cohort study. Br J Cancer 2018;118:611-5.

29. Saad M, Abdelaziz HK, Mehta JL. Aspirin for primary prevention in the elderly. Aging (Albany NY) 2019;11:6618-9. 